\title{
Nur hundertprozentige Transparenz schafft Vertrauen
}

\section{Peter Kleist}

Medizinischer Direktor GlaxoSmithKline AG

\footnotetext{
* Die Literaturangaben finden sich im Internet unter www. saez.ch $\rightarrow$ Aktuelle Nummer
} oder Archiv $\rightarrow 2013 \rightarrow 7$

Dr. med. Peter Kleist GlaxoSmithKline AG Talstrasse 3-5 CH-3053 Münchenbuchsee

peter.m.kleist[at]gsk.com
Bad Pharma? Wahrscheinlich erwarten Sie jetzt einen gewohnten Reflex: erst einmal relativieren und dementieren. So ergäben die Beispiele im Buch ein verzerrtes Bild, seien zu alt und nicht repräsentativ. Nein, das Gegenteil soll der Fall sein. Ben Goldacre, selber Arzt, ist ein Kenner der Materie und zudem ein mit mehreren Preisen ausgezeichneter Wissenschaftsjournalist. Das Buch ist sachlich geschrieben, die reichlichen Beispiele gut recherchiert. Probleme, die mit unzureichender Datentransparenz verbunden sind, werden klar dargestellt. Goldacres Werk ist kein «Schwarzbuch Pharma», das Verschwörungen aufdeckt und einzelne Unternehmen an den Pranger stellt; vielmehr geht es darum, differenziert die Missstände eines Systems anzusprechen, das falsche Anreize setzt und Fehlverhalten eher belohnt als bestraft. Neben Kritik an der Industrie findet man daher auch Kritik am Verhalten medizinischer Fachzeitschriften und der Arzneimittelbehörden.

Die Behauptung, dass Daten durch die Pharmaindustrie fabriziert oder gefälscht werden, wird man in dem Buch vergeblich suchen. Probleme ergeben sich durch die fehlende Transparenz - vor allem aufgrund einer selektiven Berichterstattung bis hin zur fehlenden Veröffentlichung ganzer Studien - die das tatsächliche Nutzen-Risiko-Verhältnis eines Arzneimittels mitunter verschleiert. Zwar verfolgen viele Unternehmen inzwischen eine rigorose Politik in Bezug auf die ausnahmslose Registrierung und Publikation ihrer Studien. Branchenweit betrachtet lassen sich Abweichungen von den Regeln einer guten Wissenschaftspraxis jedoch nicht wegdiskutieren. $\mathrm{Zu}$ viele schwarze Schafe schädigen immer noch das Ansehen einer ganzen Industrie und sorgen für ein grundsätzliches Misstrauen ihr gegenüber. Und obwohl Verstösse gegen die Publikationsstandards häufig nur subtil sind, ist es doch ihre Summe, die zu einer systematischen Verzerrung der Datenlage beiträgt und die Glaubwürdigkeit der Branche untergräbt.

Was wurde bisher unternommen? Die Verpflichtung zur Registrierung von Studien soll Druck in Richtung einer späteren, vollständigen Veröffentlichung ausüben. Doch die Effektivität von Studienregistern ist begrenzt, so lange Fachzeitschriften selbst solche mit hohem Impact Factor - weiterhin Studien publizieren, deren Auswertungen und Endpunkte deutlich von den ursprünglich gemachten
Angaben im Studienregister abweichen [1]*. Nur nebenbei bemerkt: Industriestudien schneiden dabei nicht schlechter ab als Studien aus dem akademischen Bereich [2]. Zweitens haben hohe Strafzahlungen, die einzelne Unternehmen zuletzt bei erwiesenem Fehlverhalten besonders an amerikanische Behörden leisten mussten, ihre Wirkung nicht verfehlt. Diese Unternehmen wurden zu einem Umdenken gezwungen. Doch ob der Abschreckungseffekt für andere gross genug ist, sei dahingestellt. In den meisten Ländern besteht zudem keine ausreichende gesetzliche Grundlage, um eine nur selektive Offenlegung arzneimittelbezogener Informationen spürbar zu sanktionieren. Die Reichweite der Angst vor Strafverfolgung scheint also auch nur begrenzt zu sein.

Woran fehlt es? Transparenz darf nicht als Selbstzweck betrachtet werden, nur unter Druck oder zur Schadensbegrenzung zustande kommen. Vollständige Transparenz ist vielmehr als moralischer Imperativ und berechtigter gesellschaftlicher Anspruch zu verstehen [3] - und als der einzig mögliche Weg, um die Glaubwürdigkeit des Wissenschaftssystems und die Vertrauenswürdigkeit der Industrie zu erhalten. Vollständige Transparenz wird nur dann erzielt werden, wenn die Akteure ihre Verantwortung erkennen und sich ihr stellen.

Die europäische Arzneimittelagentur EMA hat bereits zugesichert, ab 2014 die Ergebnisse der eingereichten Studien zu publizieren, sobald die Marktzulassung für ein Arzneimittel erteilt wurde (vgl. [4]). Seit Januar 2013 nimmt das British Medical Journal Publikationsmanuskripte nur noch dann an, wenn die Autoren versichern, die Originaldatensätze für weitere Forschung verfügbar zu machen [5]. Und die Industrie? Als erste Firma hat GlaxoSmithKline Ende letzten Jahres angekündigt, Zugriff auf ihre (anonymisierten) Studiendaten bis auf Patientenebene zu ermöglichen [6]. Ein Beispiel, das hoffentlich Schule macht, so dass ein moralischer Gruppendruck hin zu rückhaltloser Offenlegung der Daten aufgebaut wird. Auf jeden Fall täte die Industrie schon heute gut daran, sich von eklatantem Fehlverhalten in ihren eigenen Reihen zu distanzieren, anstatt es stillschweigend zu tolerieren.

Doch wie Ben Goldacre in einer seiner Kolumnen im Guardian bereits gesagt hat: An unseren Taten müssen wir uns letztlich messen lassen, nicht an unseren Versprechen. 


\section{Literatur}

1 Matthieu S, Boutron I, Moher D, et al. Comparison of registered and published primary outcomes in randomized controlled trials. JAMA. 2009;302:977-84.

2 Bourgeois FT, Murthy S, Mandl KD. Outcome reporting among drug trials registered in Clinical Trials.gov. Ann Intern Med. 2010;153:158-66.

3 Strech D, Littmann J. Lack of proportionality. Seven specifications of public interest that override post-approval commercial interests on limited access to clinical data. Trials. 2012;13:100.
4 Eichler HG, Abadie E, Breckenridge A, et al. Open clinical trial data for all? A view from regulators. PLoS Medicine. 2012;9:e1001202.

5 Godlee F. Clinical trial data for all drugs in current use. BMJ. 2012;345:e7304.

6 Coombes R. GlaxoSmithKline grants researchers access to clinical trial data. BMJ. 2012;345:e6909. 